University of Nebraska - Lincoln

DigitalCommons@University of Nebraska - Lincoln

$11-2007$

\title{
Bias voltage dependence of tunneling anisotropic magnetoresistance in magnetic tunnel junctions with $\mathrm{MgO}$ and $\mathrm{Al}_{2} \mathrm{O}_{3}$ tunnel barriers
}

Li Gao

IBM Research Division, Almaden Research Center, San Jose, California

Xin Jiang

IBM Research Division, Almaden Research Center, San Jose, California

See-Hun Yang

IBM Research Division, Almaden Research Center, San Jose, California

John D. Burton

University of Nebraska-Lincoln, jburton2@unl.edu

Evgeny Y. Tsymbal

University of Nebraska at Lincoln, tsymbal@unl.edu

See next page for additional authors

Follow this and additional works at: https://digitalcommons.unl.edu/physicstsymbal

Part of the Condensed Matter Physics Commons

Gao, Li; Jiang, Xin; Yang, See-Hun; Burton, John D.; Tsymbal, Evgeny Y.; and Parkin, Stuart S. P., "Bias voltage dependence of tunneling anisotropic magnetoresistance in magnetic tunnel junctions with $\mathrm{MgO}$ and $\mathrm{Al}_{2} \mathrm{O}_{3}$ tunnel barriers" (2007). Evgeny Tsymbal Publications. 7.

https://digitalcommons.unl.edu/physicstsymbal/7

This Article is brought to you for free and open access by the Research Papers in Physics and Astronomy at DigitalCommons@University of Nebraska - Lincoln. It has been accepted for inclusion in Evgeny Tsymbal Publications by an authorized administrator of DigitalCommons@University of Nebraska - Lincoln. 


\section{Authors}

Li Gao, Xin Jiang, See-Hun Yang, John D. Burton, Evgeny Y. Tsymbal, and Stuart S. P. Parkin 


\title{
Bias Voltage Dependence of Tunneling Anisotropic Magnetoresistance in Magnetic Tunnel Junctions with $\mathrm{MgO}$ and $\mathrm{Al}_{2} \mathrm{O}_{3}$ Tunnel Barriers
}

\author{
Li Gao, ${ }^{1,2}$ Xin Jiang, ${ }^{1}$ See-Hun Yang, ${ }^{1}$ J. D. Burton, ${ }^{3}$ Evgeny Y. Tsymbal, ${ }^{3}$ and Stuart S. P. Parkin ${ }^{1}$ \\ ${ }^{1}$ IBM Research Division, Almaden Research Center, San Jose, California 95120, USA \\ ${ }^{2}$ Department of Applied Physics, Stanford University, Stanford, California 94305, USA \\ ${ }^{3}$ Department of Physics and Astronomy, University of Nebraska-Lincoln, Lincoln, Nebraska 68588, USA \\ (Received 19 February 2007; revised manuscript received 2 July 2007; published 27 November 2007)
}

Tunneling anisotropic magnetoresistance (TAMR) is observed in tunnel junctions with transition metal electrodes as the moments are rotated from in-plane to out-of-plane in sufficiently large magnetic fields that the moments are nearly parallel to one another. A complex angular dependence of the tunneling resistance is found with twofold and fourfold components that vary strongly with bias voltage. Distinctly different TAMR behaviors are obtained for devices formed with highly textured crystalline $\mathrm{MgO}(001)$ and amorphous $\mathrm{Al}_{2} \mathrm{O}_{3}$ tunnel barriers. A tight-binding model shows that a fourfold angular dependence can be explained by the presence of an interface resonant state that affects the transmission of the contributing tunneling states through a spin-orbit interaction.

Magnetic tunnel junctions (MTJs) can exhibit very large changes in resistance when the relative alignment of the electrode moments is switched [1]. This tunneling magnetoresistance (TMR) is largely determined by the spin polarization of the tunneling current, which is a consequence, not simply of the spin-polarized density of states (DOS) of the ferromagnetic electrodes, but also of the tunneling matrix elements [2,3], and the spin-dependent decay of the electron wave functions across the tunnel barrier [46]. Using electrodes formed from the $3 d$ transition metal (TM) ferromagnets, such as Co-Fe alloys, and tunnel barriers formed from $\mathrm{Al}_{2} \mathrm{O}_{3}$ and $\mathrm{MgO}$, TMR values of up to $\sim 70 \%$ and $\sim 470 \%$ are observed at room temperature, respectively [5-8]. Of particular interest is the influence of the detailed electronic structure of the MTJ on the bias voltage dependence of the spin-polarized tunneling. One way to probe such an effect is to consider the angular dependence of the tunneling resistance, or the tunneling anisotropic magnetoresistance (TAMR) when the electrode's moments are rotated in large magnetic fields.

A large TAMR effect was recently observed in distinctly different MTJs formed from semiconductor heterostructures in which the ferromagnetic electrodes are formed from Mn doped GaAs [9-11]. Spin-orbit coupling (SOC) plays an important role in this material so that the TAMR effect could be attributed to a significant anisotropy in the DOS linked to the magnetization direction along different crystal axes [9-11]. Although SOC is much weaker in TMs, a small TAMR effect has been predicted for tunnel junctions with TM electrodes $[12,13]$. These calculations did not, however, consider the detailed bias or the angular dependence of the TAMR effect. In this Letter we show that MTJs with CoFe electrodes exhibit a TAMR effect, which, albeit small in magnitude, displays a complex angular dependence and has a strong dependence on the bias voltage and on the tunnel barrier material.
MTJs were fabricated on thermally oxidized Si substrates using a combination of ion-beam and magnetron sputtering at ambient temperature. A small in-plane magnetic field was applied during deposition to define the easy axis of the magnetic films. In situ shadow masks were used to pattern junctions $\sim 80 \times 80 \mu \mathrm{m}^{2}$ in area. After deposition, the devices were annealed at $260^{\circ} \mathrm{C}$ for 30 minutes in a $1 \mathrm{~T}$ field. Two types of MTJs, with $\mathrm{CoFe}$ ferromagnetic electrodes and a $\mathrm{MgO}$ or $\mathrm{Al}_{2} \mathrm{O}_{3}$ tunnel barrier, were fabricated with the following structures (from bottom to top): $100 \quad \mathrm{MgO} / 50 \quad \mathrm{Ta} / 250 \quad \mathrm{Ir}_{22} \mathrm{Mn}_{78} / 3 \quad \mathrm{Co}_{49} \mathrm{Fe}_{21} \mathrm{~B}_{30} / 60$ $\mathrm{Co}_{70} \mathrm{Fe}_{30} / 34 \mathrm{MgO} / 25 \mathrm{Co}_{70} \mathrm{Fe}_{30} / 150 \mathrm{Co}_{49} \mathrm{Fe}_{21} \mathrm{~B}_{30} / 100 \mathrm{Ta} /$ $\begin{array}{llllll}\text { and } 100 & \mathrm{TaN} / 75 & \mathrm{Ta} / 250 & \mathrm{Ir}_{24} \mathrm{Mn}_{76} / 5 & \mathrm{Co}_{49} \mathrm{Fe}_{21} \mathrm{~B}_{30} / 40\end{array}$ $\mathrm{Co}_{70} \mathrm{Fe}_{30} / 25 \quad \mathrm{Al}_{2} \mathrm{O}_{3} / 20 \mathrm{Co}_{70} \mathrm{Fe}_{30} / 100 \mathrm{Co}_{49} \mathrm{Fe}_{21} \mathrm{~B}_{30} / 50 \mathrm{Ta} /$ $75 \mathrm{Ru}$, where the numbers are nominal layer thicknesses in $\AA$.

Magnetotransport measurements, both dc and ac, were carried out at $10 \mathrm{~K}$ in a low temperature cryostat equipped with a sample rotator and a superconducting magnet. An ac lock-in technique was used to measure the dynamic resistance $R_{D}=d V / d I$ of the tunnel junctions, with a modulation amplitude of $8 \mathrm{mV} \mathrm{rms}$ at $1001 \mathrm{~Hz}$. A dc bias voltage was simultaneously applied during the $d V / d I$ measurement, with positive bias corresponding to current flowing from bottom to top. The experiment geometry was such that the magnetic field $\vec{H}$, the normal to the film plane $\vec{n}$, and the magnetic easy axis were arranged in the same plane [Fig. 1(a)]. The field direction was fixed during the experiment and the sample was rotated so that the field direction was rotated from in-plane to out-of-plane. The dc and dynamic resistances were measured, using a four-point technique, as a function of the angle $\theta$ between $\vec{H}$ and $\vec{n}$. $\theta$ values of $0^{\circ}, 180^{\circ}$, and $360^{\circ}$ correspond to magnetic fields perpendicular to the film plane, while $90^{\circ}$ and $270^{\circ}$ correspond to fields along the in-plane easy axis direction. 
(a)

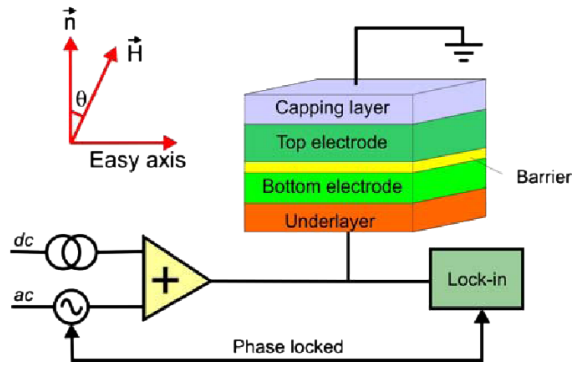

(b)

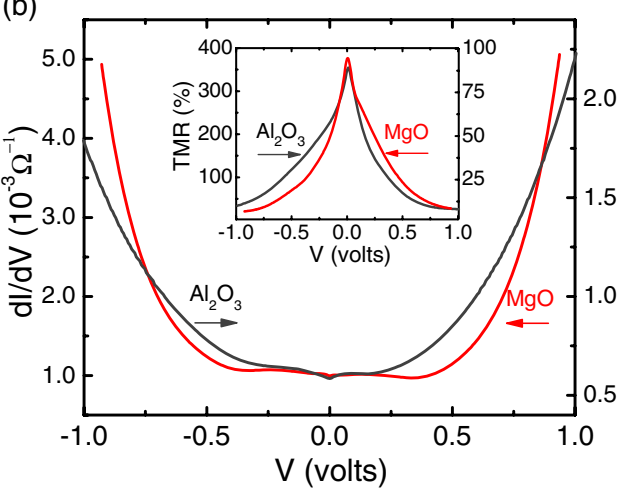

FIG. 1 (color online). Schematic diagram of the TAMR measurement setup (a) and the bias dependence of differential conductance for the $\mathrm{MgO}$ and $\mathrm{Al}_{2} \mathrm{O}_{3}$ MTJs in a perpendicular field of $7 \mathrm{~T}$ at $10 \mathrm{~K}$ (b). The inset in (b) shows the bias dependence of the differential TMR at $10 \mathrm{~K}$.

Figure 1(b) depicts the differential conductance $d I / d V$ as a function of bias voltage at $10 \mathrm{~K}$ in a perpendicular field of $7 \mathrm{~T}\left(\theta=0^{\circ}\right)$, for MTJs with $\mathrm{Al}_{2} \mathrm{O}_{3}$ and $\mathrm{MgO}$ tunnel barriers, respectively. Data at $\theta=90^{\circ}$ closely resemble those at $\theta=0^{\circ}$. The inset in Fig. 1(b) shows the corresponding bias dependence of the differential TMR, defined as $\left[(d I / d V)_{P}-(d I / d V)_{\mathrm{AP}}\right] /(d I / d V)_{\mathrm{AP}}$. Here $d I / d V$ is measured in an in-plane field; $P$ and AP stand for parallel and antiparallel alignment of the CoFe moments, respectively. Zero-bias TMR values of $377 \%$ and $89 \%$ were obtained for the MTJs with $\mathrm{MgO}$ and $\mathrm{Al}_{2} \mathrm{O}_{3}$ barriers, respectively, indicating the high quality of these devices.

$R_{D}$ vs $\theta$ curves at various bias voltages are plotted in Fig. 2(a) for the $\mathrm{MgO}$ junction. The measurements were taken at $10 \mathrm{~K}$ in a field of $7 \mathrm{~T}$, which is sufficiently large to almost fully saturate the magnetization of the CoFe electrodes parallel to the field. The junction resistance is normalized to its average value over $\theta$ at each bias voltage. At low bias, the $R_{D}$ vs $\theta$ curves are twofold symmetric, with peaks at $\theta=0^{\circ}, 180^{\circ}, 360^{\circ}$, and valleys at $\theta=90^{\circ}, 270^{\circ}$. As the bias is increased, the valleys near $\theta=90^{\circ}, 270^{\circ}$ broaden and, eventually, a second set of peaks appears for bias voltages exceeding $\sim-0.4 \mathrm{~V}$ or $+0.45 \mathrm{~V}$. This suggests that an additional component with fourfold symmetry contributes to TAMR at high bias. The magnitude of TAMR is fairly symmetric with respect to bias polarity at low bias. At high bias, however, TAMR is much smaller for positive bias.
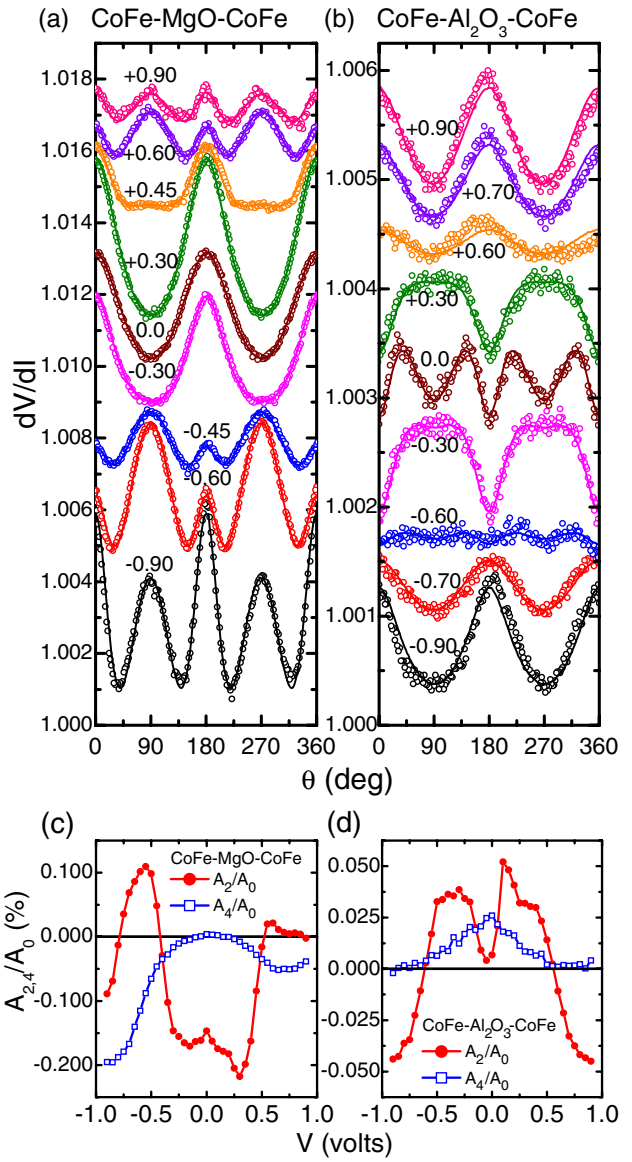

FIG. 2 (color online). Normalized $R_{D}$ vs $\theta$ curves (symbols) at various bias voltages for $\mathrm{MgO}$ (a) and $\mathrm{Al}_{2} \mathrm{O}_{3}$ (b) MTJs. The data are displaced vertically for clarity. The solid lines are fits using Eqs. (1) and (2). (c),(d) The fitting parameters $A_{2} / A_{0}$ (solid circles) and $A_{4} / A_{0}$ (open squares) as a function of bias for the $\mathrm{MgO}$ and $\mathrm{Al}_{2} \mathrm{O}_{3}$ MTJs.

In Figs. 3(a) and 3(c), the normalized junction resistance is plotted as a function of bias and angle, for the dynamic and $\mathrm{dc}$ resistance measurements, respectively. The data were taken, in each case, every $2^{\circ}$ and every $50 \mathrm{mV}$ (note that in the $\mathrm{dc}$ case no data are possible at zero bias). The contrast in the contour plot represents the magnitude of the normalized junction resistance. The dc and dynamic resistances measure different quantities. The former integrates contributions from electrons distributed over a wide energy range up to the bias voltage, whereas the latter is sensitive to tunneling in a narrow energy range, determined by the modulation amplitude (here, $\sim 24 \mathrm{mV}$, peak to peak). The ac measurement clearly accentuates the dependence of the TAMR effect on both voltage and angle, as shown in Fig. 3. For this reason, we focus our discussion on the dynamic resistance data.

For the $\mathrm{MgO}$ barrier, $R_{D}$ shows valleys at $\theta=90^{\circ}, 270^{\circ}$ at low bias, which are seen as dark areas in Fig. 3(a). The emergence of a second set of peaks can be clearly distinguished above a threshold voltage of $\sim-0.4 \mathrm{~V}$ and $+0.45 \mathrm{~V}$, for negative and positive voltages, respectively. 


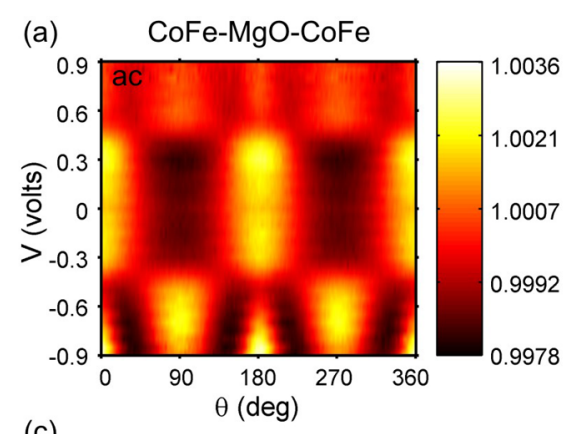

(c)

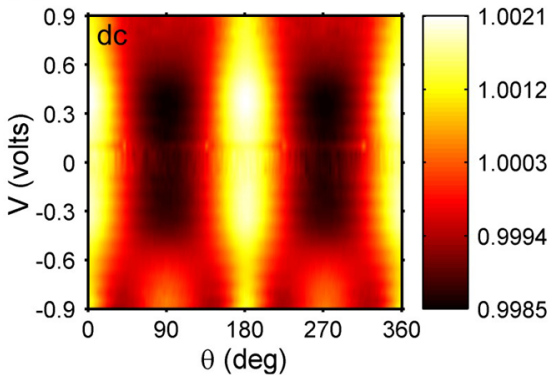

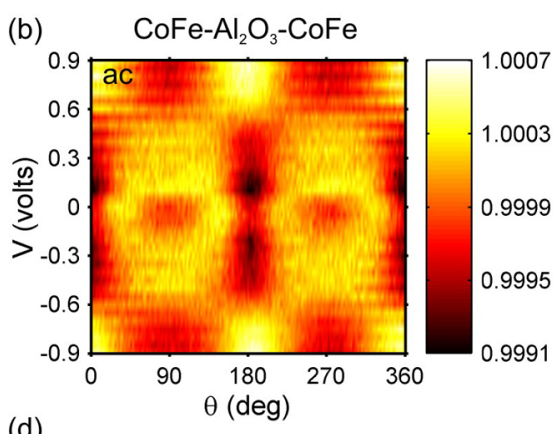

(d)

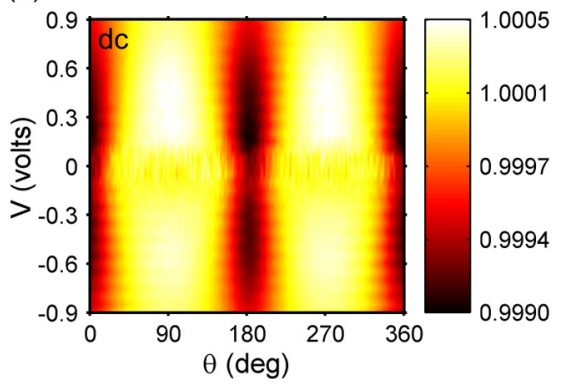

FIG. 3 (color online). (a),(b) Contour plots of $R_{D}$ as a function of bias and angle for the $\mathrm{MgO}$ and $\mathrm{Al}_{2} \mathrm{O}_{3}$ MTJs. (c),(d) The corresponding contour plots of dc resistance.
Note that this distinctive change in the angular dependence is obscured in the dc resistance plot. Above this threshold voltage the angular dependence shows clear evidence for some fourfold character.

The TAMR effect of the $\mathrm{Al}_{2} \mathrm{O}_{3}$ junction has distinctly different characteristics compared with that of the $\mathrm{MgO}$ junction, as shown in Figs. 2(b), 3(b), and 3(d). Generally speaking, the TAMR magnitude is much smaller than that of the $\mathrm{MgO}$ junction. In the low bias regime, the $R_{D}$ vs $\theta$ curve contains a fourfold symmetric component with minima at $\theta=0^{\circ}$ and $90^{\circ}$. As the bias increases, the curve becomes largely twofold symmetric. Below $\sim \pm 0.6 \mathrm{~V}$, the curve has a maximum at $\theta=90^{\circ}$ and a minimum at $\theta=$ $0^{\circ}$. Above $\sim \pm 0.6 \mathrm{~V}$, however, the positions for the resistance extrema are reversed; i.e., the maximum is at $\theta=$ $0^{\circ}$ and the minimum is at $\theta=90^{\circ}$. These features are clearly seen in Fig. 3(b). Note that the basic characteristics of the contour plots shown in Fig. 3 do not change with field for the field range explored (from 5 to $9 \mathrm{~T}$ ).

To check that the observed TAMR results from spindependent tunneling, we fabricated tunnel junctions with nonmagnetic aluminum electrodes with the following structure: $100 \mathrm{MgO} / 150 \mathrm{Al} / 32 \mathrm{MgO} / 150 \mathrm{Al} / 100 \mathrm{Ta}$ (layer thicknesses in $\AA$ ). No TAMR was measured within the experimental noise level for these control samples.

The $R_{D}$ vs $\theta$ curve at a given bias can be fitted using the following equations:

$$
\begin{gathered}
R_{D}(\theta)=\left(A_{0}+A_{2} \cos 2 \phi+A_{4} \cos 4 \phi\right)^{-1}, \\
\frac{\cos \phi}{\sin \phi}=\frac{H \cos \theta-4 \pi M_{s} \cos \phi}{H \sin \theta},
\end{gathered}
$$

where $\phi$ is the angle between the CoFe magnetization direction and the film normal direction $\vec{n}$ after taking into account the demagnetization field; $M_{s}$ is the saturation magnetization of $\mathrm{CoFe} ; A_{0}, A_{2}$, and $A_{4}$ are fitting parameters. The ratios $A_{2} / A_{0}$ and $A_{4} / A_{0}$ can be understood as half of the TAMR magnitude of the components with twofold and fourfold symmetries, respectively. Their signs are related to the positions of the resistance extrema in the $R_{D}$ vs $\theta$ curves. Assuming $4 \pi M_{S}=1.93 \mathrm{~T}$, excellent agreement between data and fits are obtained [see Figs. 2(a) and 2(b)]. $A_{2} / A_{0}$ and $A_{4} / A_{0}$ values, obtained from the fits, are plotted in Figs. 2(c) and 2(d), which show very complicated bias dependences for both types of junctions.

The TAMR effect likely originates from SOC, which gives rise to an anisotropy of the DOS of the bulk electrodes $[9,10,12]$ and that of their interfaces [13] with respect to the magnetization direction. The pronounced difference between the $\mathrm{MgO}$ and $\mathrm{Al}_{2} \mathrm{O}_{3}$ MTJs points to the interface electronic structure as the origin of the TAMR.

Using a simple tight-binding model, we demonstrate that the influence of resonant states on the interface DOS of the majority band can lead to the observed evolution of TAMR from a twofold to a fourfold angular dependence. Note that we have examined the band structure of bcc CoFe along the $\Gamma-H\left(k_{\|}=0\right)$ direction in the Brillouin zone. Specifically, we explored the lifting of degeneracy in the bulk band structure due to SOC and found no fourfold component in the angular dependence of the DOS at any energy sufficient to lead to the observed variation in the experiments. This suggests that the fourfold component does not arise from the bulk band structure of CoFe.

We model the $\Delta_{1}$ majority-spin band in bec CoFe [4] by a one-dimensional tight-binding band. We consider the case when this band is coupled to a minority resonant state localized at the interface via SOC described by the parameter $\lambda$. The anisotropy in the conductance is determined by the anisotropy of the interface DOS of the majority $\Delta_{1}$ band, which can be found from the corre- 


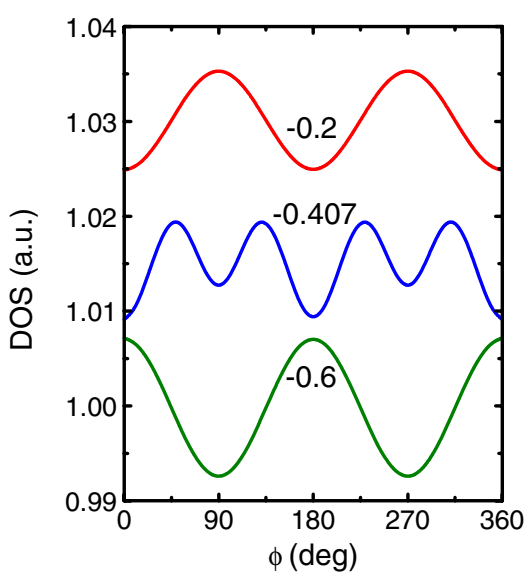

FIG. 4 (color online). Angular dependence of the interface DOS of the majority band (in arbitrary units) for several energies $E$ near the resonant energy $E_{r}=-0.4 \mathrm{eV}$. Energies are given in $\mathrm{eV}$. The majority band has width $4 \mathrm{eV}$ and is centered at $0.6 \mathrm{eV}$. The width of the resonant state $\gamma_{0}=10 \mathrm{meV}$, and the spin-orbit coupling parameter $\lambda=50 \mathrm{meV}$.

sponding interface Green's function, $g(E)$. We note that, due to the weak energy dependence of the $\Delta_{1}$ electron band's decay function through a crystalline $\mathrm{MgO}$ barrier [4], states far below $E_{F}$ can significantly contribute to the tunneling current, contrary to the free electron band case [14]. By solving Dyson's equation we find that

$$
g(E)=\frac{g_{0}(E)}{1-\lambda^{2} g_{0}(E) g_{r}(E) \cos ^{2} \phi},
$$

where $g_{0}(E)$ is the interface Green's function of the majority band in the absence of SOC [15] and $g_{r}(E)=(E-$ $\left.E_{r}+i \gamma_{0}\right)^{-1}$ is the Green's function of the resonant level with $E_{r}$ and $\gamma_{0}$ being the resonance energy and width, respectively.

In Fig. 4 we plot the interface DOS of the majority band, $\rho=-\operatorname{Im}(g) / \pi$, as a function of $\phi$ for several energies near the resonant level which we have chosen to lie at $E_{r}=$ $-0.4 \mathrm{eV}$. Away from the resonance the angular dependence of the interface DOS has a twofold symmetry. This twofold angular dependence changes sign near the resonant energy. Associated with this sign change is the onset of a significant fourfold angular variation. Since the tunneling current will largely be determined by the interface DOS of the majority $\Delta_{1}$ channel, this same variation will appear in the $R_{D}$ measurement when the window of applied bias passes the resonance.

It is known that the bcc $\mathrm{Fe}(001)$ surface supports a minority-spin surface state [16]. The relevant interface state is present in $\mathrm{Fe}(001) / \mathrm{MgO} / \mathrm{Fe}$ tunnel junctions, as indicated by theory $[4,15]$ and experiment [17]. A similar resonant state is likely present at the bcc $\mathrm{CoFe}(001)$ surface and the $\mathrm{CoFe}(001) / \mathrm{MgO}$ interface. Using a rigid band model to estimate the change in the Fermi energy due to the increase in valence of $\mathrm{CoFe}$ as compared to $\mathrm{Fe}$, we find the position of the interface resonant state for $\mathrm{Co}_{70} \mathrm{Fe}_{30}(001) / \mathrm{MgO}$ interface is $\sim 0.4 \mathrm{eV}$ below the Fermi energy. This is consistent with the observation of significant fourfold symmetry for bias beyond $\sim-0.4 \mathrm{~V}$ or $+0.45 \mathrm{~V}$ for $\mathrm{MgO}$ based MTJs, as shown in Fig. 2 .

In the case of $\mathrm{Al}_{2} \mathrm{O}_{3}$ based MTJs a similar effect may occur due to the possibility of a narrow majority interface resonant band derived from excess oxygen at the interface [15]. It is predicted that this band lies close to the Fermi level and is strongly transmitting. An analysis based on a model similar to that above reveals that an interface resonant DOS also has a significant fourfold angular variation when coupled to a bulk band via SOC. This is consistent with the observation of a strong fourfold dependence of the variation observed in $\mathrm{Al}_{2} \mathrm{O}_{3}$ at low bias in Fig. 2.

In conclusion, we have observed a tunneling anisotropic magnetoresistance effect in magnetic tunnel junctions with $3 d$ transition metal ferromagnetic electrodes for both crystalline and amorphous tunnel barriers, despite the weak spin-orbit coupling in these systems. We find complex dependences of the junction resistance on the bias voltage and angle, which are distinctly different for $\mathrm{MgO}$ and $\mathrm{Al}_{2} \mathrm{O}_{3}$ tunnel barriers. A tight-binding model suggests that the TAMR effect derives from the anisotropy in the interface density of states of the majority band due to mixing with a resonant state via spin-orbit coupling.

We thank Professor W. Harrison, Dr. M. Chshiev, and Professor W. Butler for stimulating discussions. This project is partially supported by the DMEA. The research at UNL is partially supported by NSF-MRSEC.

[1] S. S. P. Parkin et al., Proc. IEEE 91, 661 (2003).

[2] E. Y. Tsymbal and D. G. Pettifor, J. Phys. Condens. Matter 9, L411 (1997).

[3] C. Kaiser et al., Phys. Rev. Lett. 94, 247203 (2005).

[4] W. H. Butler et al., Phys. Rev. B 63, 054416 (2001).

[5] S. S. P. Parkin et al., Nature Mater. 3, 862 (2004).

[6] S. Yuasa et al., Nature Mater. 3, 868 (2004).

[7] J. J. Yang et al., Appl. Phys. Lett. 89, 202502 (2006).

[8] J. Hayakawa et al., Appl. Phys. Lett. 89, 232510 (2006).

[9] C. Gould et al., Phys. Rev. Lett. 93, 117203 (2004).

[10] C. Rüster et al., Phys. Rev. Lett. 94, 027203 (2005).

[11] H. Saito, S. Yuasa, and K. Ando, Phys. Rev. Lett. 95, 086604 (2005).

[12] A. B. Shick et al., Phys. Rev. B 73, 024418 (2006).

[13] A. N. Chantis et al., Phys. Rev. Lett. 98, 046601 (2007).

[14] The weak energy dependence of the electron's decay function is due not only to the particular band structure of $\mathrm{MgO}$ but also to the Fermi level lying near the band gap center. If the Fermi level were close to either the conduction or the valence band, the variation of the decay constant with energy could not be ignored.

[15] E. Y. Tsymbal et al., Prog. Mater. Sci. 52, 401 (2007).

[16] J. A. Stroscio et al., Phys. Rev. Lett. 75, 2960 (1995).

[17] C. Tiusan et al., Phys. Rev. Lett. 93, 106602 (2004). 